\title{
Solid Electrolyte Interphase instability in operating Lithium-ion bat- teries unraveled by Enhanced-Raman Spectroscopy
}

\author{
Antonin Gajan, ${ }^{1}$ Constance Lecourt, ${ }^{1}$ Blanca Torres Bautista, ${ }^{1}$ Laure Fillaud, ${ }^{1}$ Julien \\ Demeaux, ${ }^{2}$ and Ivan T. Lucas, ${ }^{1,3}$ \\ ${ }^{1}$ Sorbonne Université, CNRS, Laboratoire LISE, 75000 Paris, France \\ ${ }^{2}$ Saft, Corporate Research, 33074, Bordeaux, France \\ ${ }^{3}$ Lead Contact
}

\begin{abstract}
The fundamental understanding of the electrode/electrolyte interfacial processes in lithium-sodium ion batteries (LIBs) and of their dynamics upon cycling is of prime importance for the development of new generation electrode materials. Operando analyses using the utmost sensitive techniques are required to produce an accurate depiction of the underlying processes at the origin of the battery performance decay. If enhanced Raman Spectroscopy through the use of signal nano-amplifiers (SHINs) shows the required sensitivity, its implementation in operando conditions and particularly on functional materials in contact with organic electrolytes remains challenging. This work through extensive optimization of SHINERS conditions for operando diagnosis of $L I B$ materials, including the design of near-infrared active amplifiers and the control of the photon dose, demonstrates the possibility to track the dynamics of composition of the electrode/electrolyte interface upon cycling of $L I B$ coin-cells and uncovers the origin of the irreversible capacity of tin electrodes proposed as alternative to

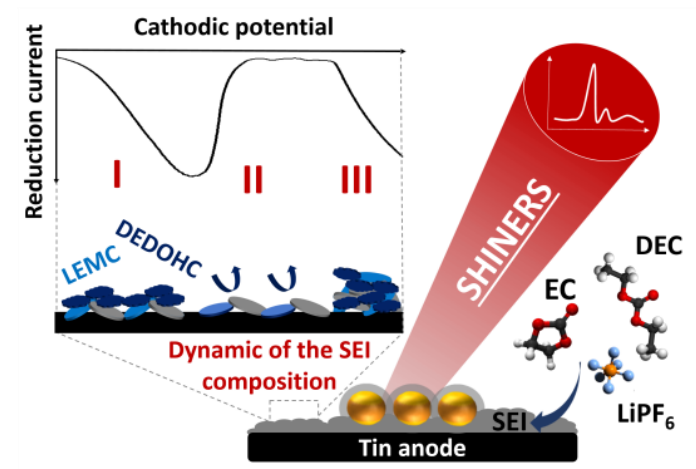
graphite anodes.
\end{abstract}

New high-energy electrode materials proposed as alternative to graphite anode (i.e. tin, silicon) and layered cathode materials hold great promise for the development of new generation of lithium-ion batteries ${ }^{1}(L I B \mathrm{~s})$, but the ineluctable degradation of their performance during the repeated charge and discharge cycles still limits their wide scale development. One key problem lays at the electrode-electrolyte interface, where non-aqueous electrolyte reacts at the electrode surface upon charging of the battery. Reactions products either diffuse away or accumulate at the electrode surface to form semi-passive layers, the Solid Electrolyte Interphase $S E I^{2}$ on the anode side and the Cathode Electrolyte Interphase $C E I I^{3}$ The properties of such surface layers (stability/build-up, ionic diffusivity, passivating ability) are mainly dictated by the composition of the electrolyte (reduction and oxidation potential) and by the nature of the electrode material (intrinsic reactivity, lithiation mechanism). ${ }^{4}$ To minimize the performance decay of newly developed $L I B$ electrode materials, both the electrolyte and the electrode formulation should be therefore revisited. ${ }^{5}$

Much attention has been paid these last four decades to the processes taking place at the anodes with a focus on the SEI properties $^{678}$ and more recently on the $C E I .{ }^{9}$ One main challenge associated to the characterization of surface films developed on electrode materials remains the composition evaluation, especially in operando conditions. The characterization of thin passive films (tens of nanometer thick) is not that straightforward as very few techniques possess the sufficient sensitivity, or provide clear/meaningful chemical information with limited interference with the probe (photon- or electron-beam damage associated to $\mathrm{x}$-ray or electron spectroscopies). Extensive efforts to access in situ / operando composition characterization of electrode material surface in contact with liquid electrolyte have been deployed, with techniques like $F T I R^{1011}$, differential electrochemical mass spectrometry $D E M S^{12}$, nonlinear-spectroscopy (Sum Frequency Generation: $S F G)^{13}$ and more recently Raman spectroscopy. Raman spectroscopy, although powerful to characterize electrode materials ${ }^{14}$, shows blind when it comes to characterize thin surface layers like SEI made of (in)organic compounds with low cross-section for Raman scattering. To circumvent this limitation, Raman signal enhancement techniques using the plasmon resonance properties of metal nanostructures ${ }^{15}$ were successfully implemented to extract compositions of surface films developed on various electrode materials upon contact with organic carbonate electrolytes. Surface Enhanced Raman spectroscopy (SERS) was implemented operando on SERS-active silver and gold nanostructured electrodes starting from 2000. ${ }^{16} 1718$ More recently Tipenhanced Raman Spectroscopy (TERS) using silver or gold scanning nanoprobes demonstrated the ex situ mapping of the SEI composition distribution with nanoscale resolution on a 
non-SERS active electrode material (silicon anode ${ }^{19}$ ) in a similar way as nano-infrared mapping was achieved on tin anodes a few years before. ${ }^{20}$ TERS, despite recent significant advances since its demonstration under operando conditions ${ }^{21} 22$, has not been yet implemented in $L I B$ electrolyte, due to the difficulty to control the atmosphere and prevent the electrolyte evaporation in open cells. Alternatively, Shell-Isolated Nanoparticle-Enhanced Raman Spectroscopy $(\text { SHINERS })^{23}$ using metal@ $\mathrm{SiO}_{2}$ core-shell nanoparticles as plasmonic signal amplifiers deposited on pristine electrode materials has been carried out to study chemical changes at various electrode/electrolyte interfaces upon cycling in $L I B$ electrolyte, including silicon ${ }^{24}$ and carboncoated $\mathrm{Zn}_{0.9} \mathrm{Fe}_{0.1} \mathrm{O}$ anodes ${ }^{25}$ and $\mathrm{LiNi}_{x} \mathrm{Mn}_{y} \mathrm{Co}_{1-x-y} \mathrm{O}_{2}$ (NMC) cathodes. $^{26}$

In this study, we have addressed three major gaps in analytical studies of interfacial processes in $L I B$ electrolyte using enhanced Raman techniques: $i$ ) the usual misconception of in situ (spectro-electrochemical) cells, $i i$ ) the possible interference of the Raman laser and light nano-amplifiers with beam-sensitive organic electrolytes ("knock-out" damages), iii) the inaccurate interpretation of SEI/CEI chemical signatures based on infrared spectra or computed Raman spectra $(D F T)$. By revisiting the SHINERS implementation under operando conditions, we were able to track the dynamics of interfacial composition changes on tin anodes upon cycling in LIB electrolyte. This work provides important insights in the degradation mechanism of organic carbonate electrolytes and on the origin of the interfacial instability specific to tin, ${ }^{27}$ proposed as an alternative to graphite given its high theoretical lithium retention capability (> 900 mAh.g $\left.{ }^{-1}\right)$.

SHINERS optimization in LIBs - SHINs decorated tin electrodes, polypropylene thin separator and lithium foil counterelectrode were assembled in a coin-cell like operando Raman cell (Figure 1a) filled with minimum amounts of electrolyte $(60 \mu \mathrm{L}$, preventing from further re-dissolution of the SEI, as discussed in Supplementary Information (SI, Fig. S4). Au@ $\mathrm{SiO}_{2}$ nano-bipyramid SHINs were synthesized by adapting two multistep protocols described by Liz-Marzan ${ }^{29}$ and Guyot-Sionnest $^{30}$ and then drop-casted on the pristine tin electrode. The association of such anisotropic SHINs with near-infrared laser sources $(785 \mathrm{~nm})$, provides the highest Raman signal enhancement while minimizing the strong fluorescence signal of battery electrolytes and cycled electrodes, allowing the extraction of the Raman signal of a molecular monolayer in contact with $L I B$ electrolytes (SI, Fig. S5). We introduced here also for the first time the use of scanning Raman probe $(50 \mu \mathrm{m}$ diameter circular pattern) for SHINERS to prevent electrolyte degradation inherent to photothermal effect or possible light-induced electron transfer (plasmon electrochemistry ${ }^{31}$ ) at the electrode/electrolyte interface. In the scanning mode, the photon dose per surface unit can be considerably reduced while maintaining high level of Raman signal, as demonstrated by the untouched electrolyte signature obtained even after $200 \mathrm{~s}$ at $4.5 \mathrm{~mW}$ (see Figure 1b). The full description of the revisited SHINERS implementation can be found in SI (Fig. S2,4,5).

Dynamics of the interfacial composition on tin - Operando SHINERS measurements were then performed every $100 \mathrm{mV}$ (chronoamperometry step, $300 \mathrm{~s}$ ) starting from the open circuit potential $2.8 \mathrm{~V}(O C P)$ down to $100 \mathrm{mV}$ (two forward scans separated by $20^{\prime}$ at the $O C P$ to let the electrode to relax). $E C-D E C$ $L i P F_{6}$ electrolyte mixture was chosen for its poor filming ability on tin anodes described in previous works. ${ }^{32} \mathrm{~A}$ typical cyclic voltammetry $\left(C V, 1 \mathrm{mV} . \mathrm{s}^{-1}\right)$ of the tin electrode in such electrolyte (Figure 1a) shows a strong intensity reduction peak centered on $1.0 \mathrm{~V}$ in addition to the lithiation/delithiation current peaks at potential lower than $0.4 \mathrm{~V}$ and higher than $0.7 \mathrm{~V}$ respectively. In contrast with $E C-E M C L_{i P F_{6}}$ electrolyte mixture (further discussed in SI, Fig. S4), the passivation of tin is not achieved after the first cycle as the irreversible peak at $1.0 \mathrm{~V}$ is still of strong intensity during the second $C V$ scan.
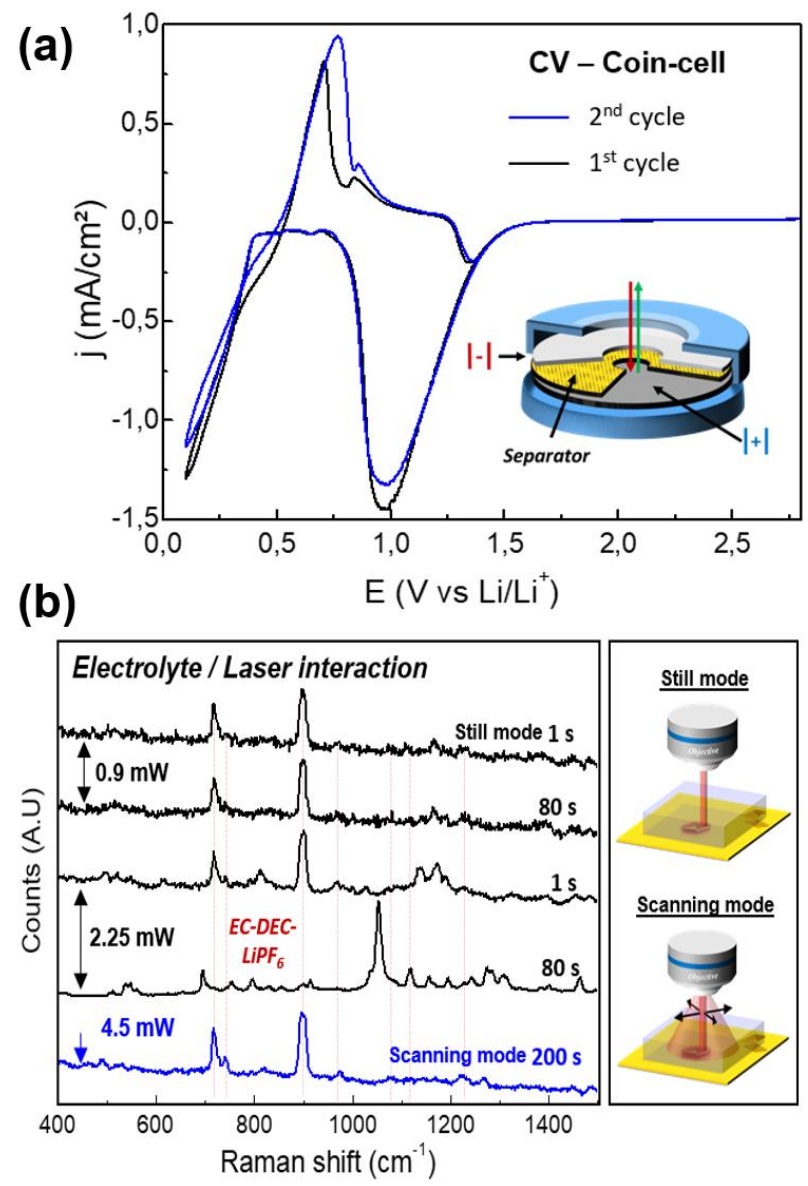

Figure 1: (a) Electrochemical response (cyclic voltammetry) of a tin electrode in the spectro-electrochemical coin-cell filled with EC-DEC LiPFo electrolyte (counter electrode: lithium foil). (b) Electrolyte Raman signature depending on the time of exposure to the laser probe, on the laser power and on the signal excitation mode ("still" laser or "scanning" laser).

Selected individual spectra and 2D color map (spectra $v s$ potential) corresponding to the first (forward) potential scan are presented in Figures $2 \mathbf{a} \boldsymbol{\&} \mathbf{b}$ respectively. For the sake of clarity, only spectra associated to the $2 \mathrm{~V}-0.1 \mathrm{~V}$ potential range are presented (the full set of data can be found in SI, Fig. S8). Spectra were baseline subtracted and normalized to the intensity of the electrolyte band at $900 \mathrm{~cm}^{-1}$. The first potential forward scan extracted from the $C V$ cycling of tin (Figure 1a) in such electrolyte at $1 \mathrm{mV} . \mathrm{s}^{-1}$ is given to ease the correlation between the electrochemical phenomena and the chemical changes at a given applied potential. While between the $O C P$ and $2 \mathrm{~V}$ no Raman bands other than the ones of the electrolyte are detected (current intensity close to zero), a few noticeable spectral changes are visible at potential as low as $1.6 \mathrm{~V}$, which corresponds to the onset of the reduction current peak centered at $1 \mathrm{~V}$ on tin electrode:

- starting from $1.6 \mathrm{~V}$, new features at 501, 1065/1074, 1139, $1266 / 1285,1486 \& 1573 \mathrm{~cm}^{-1}$ highlighted by black and red dotted lines emerge,

- while the intensity of some bands remains constant throughout the whole remaining potential range, i.e. $600,1139,1486$ 

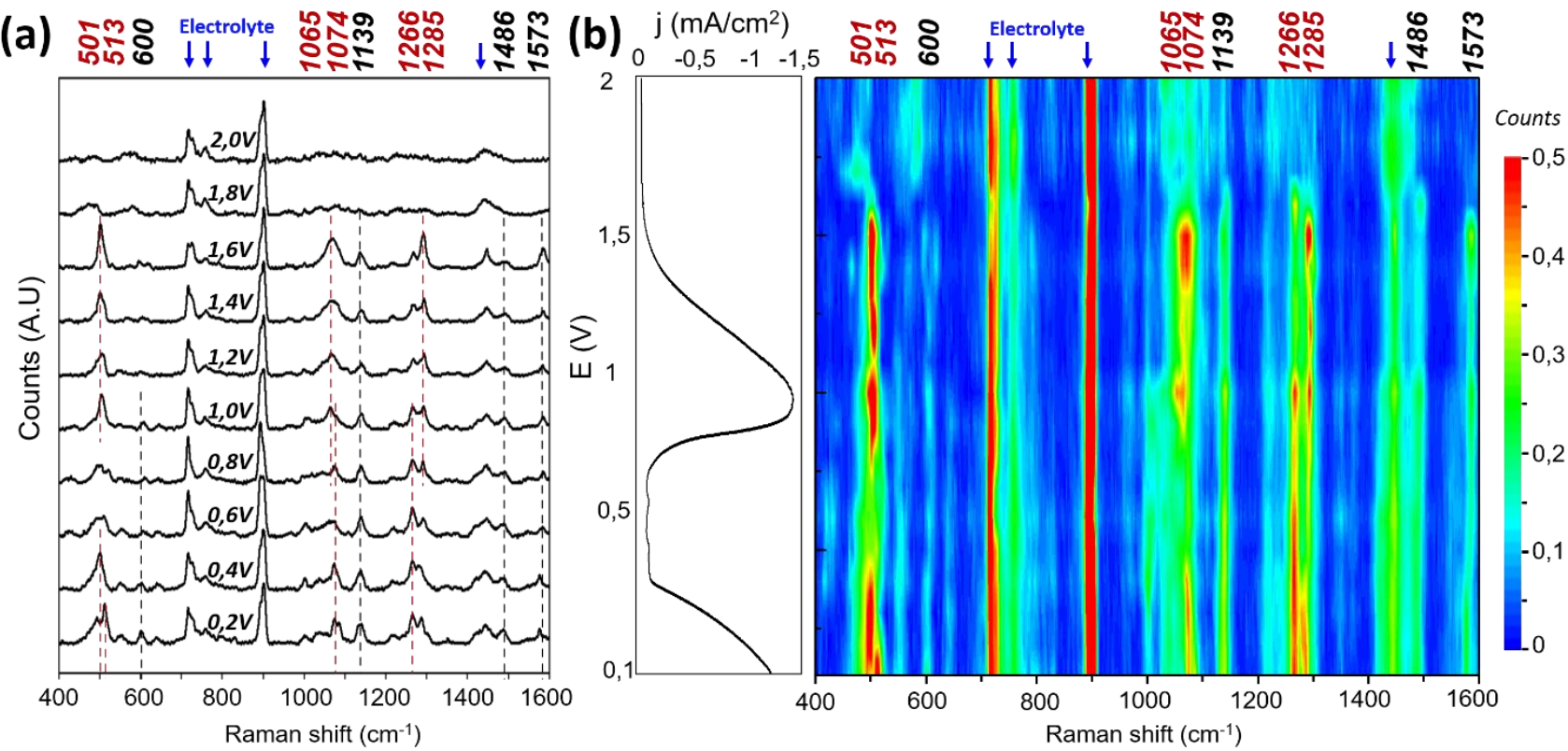

Figure 2: Dynamics of the SEI composition on tin in EC-DEC LiPF - (a) Operando SHINERS on tin electrode polarized in $E C / D E C=50 / 50(\mathrm{v} / \mathrm{v}), 1.0 \mathrm{M} \mathrm{LiPF} 6$ electrolyte. Raman spectra were collected at $785 \mathrm{~nm}$ (laser power $0.9 \mathrm{~mW}$, tacq $=3$ seconds, 8 accumulations) every $100 \mathrm{mV}$ steps (after 5 min chronoamperometry) from the OCP $(2,8 \mathrm{~V})$ down to $0.1 \mathrm{~V} v \mathrm{si} / \mathrm{Li}+$ (first forward scan). Dotted lines correspond to new Raman bands appearing during polarization (only bands marked with red dotted lines were attributed). (b) A linear sweep voltammogram obtained on this same system between $2.0 \mathrm{~V}$ and $0.1 \mathrm{~V}$ (versus lithium) at $1 \mathrm{mV} / \mathrm{s}$ is given to guide the reader eyes. The $2 \mathrm{D}$ color map representing the Raman band intensity as a function of the applied potential allows to appreciate the dynamics of composition of the tin electrode lelectrolyte interface.

and $1573 \mathrm{~cm}^{-1}$, the bands at 501, 1065/1074, 1266/1285 $\mathrm{cm}^{-1}$ see marked changes in intensity in the $1-0,4 \mathrm{~V}$ potential range, when the current associated to this irreversible peak falls to zero and the lithiation has not yet started. Interestingly, the 501, $1065 / 1074$ and $1285 \mathrm{~cm}^{-1}$ band intensities fade when the one at 1266 increases,

- upon lithiation, a new band at $513 \mathrm{~cm}^{-1}$ appears, the band at $1065 \mathrm{~cm}^{-1}$ remains constant and the bands at 501, 1074 and 1285 $\mathrm{cm}^{-1}$ increase in intensity. Similar behavior is observed during the second forward scan as depicted in Figure 3, with the bands at 501 and $513 \mathrm{~cm}^{-1}$ which increase concomitantly with the irreversible current peak and momentary disappears in the $1-0.4 \mathrm{~V}$

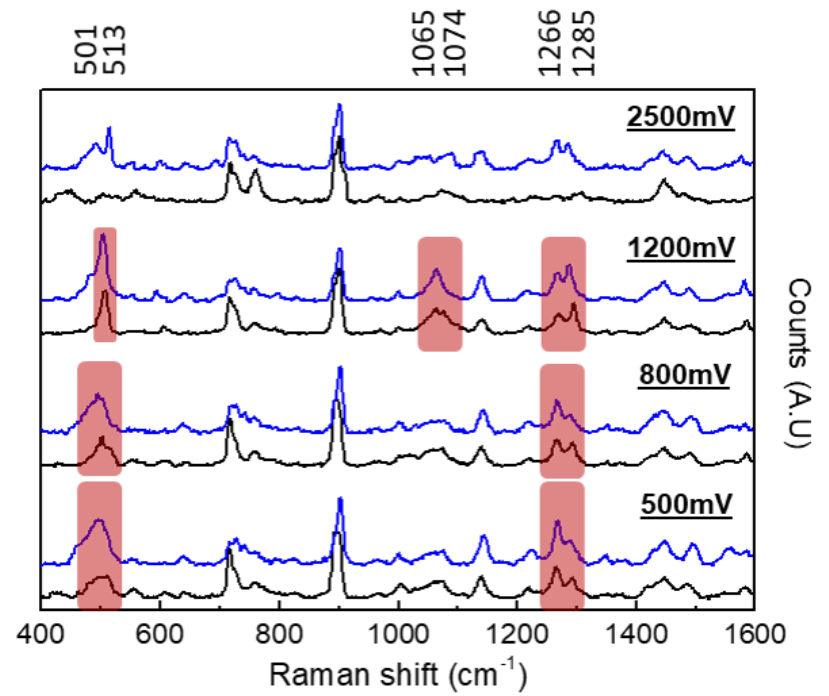

Figure 3: Comparison of SHINERS spectra collected on tin in EC/DEC LiPF 6 electrolyte during the second (blue) and first (black) forward potential scans, the bands of interest are enlightened in red. potential range. Also, the $1065 / 1074$ and $1285 \mathrm{~cm}^{-1}$ band intensities increase and then fade while the band at $1266 \mathrm{~cm}^{-1}$ increases. Note that no changes in the Raman signatures were observed upon relaxation of the electrode potential to the $O C P$ in between the two forward scans.

The successful tracking of the SEI composition upon cycling, despite the fact that the $S E I$ thickness can reach on tin electrodes tens of nanometers ${ }^{34}$ (Raman signal from gold bipyramids through such a thick dielectric layer could be compromised), can be explained by the fact that the SEI builds up only at the electrode surface with the SHINs appearing as floating on the surface film developed on cycled and rinsed tin electrodes (Figure 4b). If operando SHINERS tests run on tin anodes in polypropylene carbonate (PC)-based electrolyte were also successful (SI, Fig. S17, full results to be published), loss of Raman signal on tin was systematically observed at potential lower than $1 \mathrm{~V}$ vs $\mathrm{Li} / \mathrm{Li}^{+}$in $E C$-EMC $\mathrm{LiPF}_{6}$ (SI, Fig. S10) and could not be recovered upon scanning at higher potential. Further work is necessary to understand the relation between the filming properties of the electrode depending on the electrode material and on the electrolyte, the potential applied and the behavior of the plasmon signal amplifiers.

SEI SHINERS signatures assignment - The recurrent problem of spectral assignment was addressed here by establishing a databank of Raman signatures of a series of organic compounds reported in the literature ${ }^{48}{ }^{8}$ as possible degradation products of carbonate based electrolytes. These latter were either purchased ( $\mathrm{DEDOHC}, \mathrm{Li}_{2} \mathrm{CO}_{3}, \mathrm{LiF}, \mathrm{PEO}$, sodium propionate) or synthesized (LEC, LMC, LEMC, LEDC, LPDC) through much effort and precautions by adapting different published protocols detailed in SI. A series of complementary operando experiments (tin in $P C-L i P F_{6}$, gold in $E C-D E C L i P F_{6}$ ) or $e x$ situ tests were also carried out to emphasize the strong dependence of the SEI composition with the nature of the electrode material and of the electrolyte and the pertinence/robustness of the present analysis. 
By comparison of spectra presented in Figure 4a and in SI (Fig. S11,S13,S14,S15,S16) we propose the following band assignment:

- the double $501-513 \mathrm{~cm}^{-1}$ band which is of strong intensity on tin in situ, almost absent ex situ on rinsed electrode, could be assigned to Diethyl 2,5-Dioxahexane Dicarboxylate (DEDOHC), as this compounds is liquid at room temperature and soluble in the electrolyte. Note that this band is absent on gold (SI, Fig. S7).

- the bands at 1074 and $1266 \mathrm{~cm}^{-1}$ (which is the most pronounced feature on rinsed tin electrode and which also appears on gold) could be assigned to lithium ethylene monocarbonate (LEMC).

$\boldsymbol{L E M C}$ vs $\boldsymbol{L E D C}$ - Lithium Ethylene dicarbonate (LEDC) has been proposed as early as 1996 by Aurbach's group as a possible product of reduction of ethylene carbonate $(E C)$ on noble metal electrodes $(N i, A u)$ via a single electron pathway ${ }^{33} 34$ and since has been identified in situ by ATR on gold electrode ${ }^{4}{ }^{10}$ by

(a)

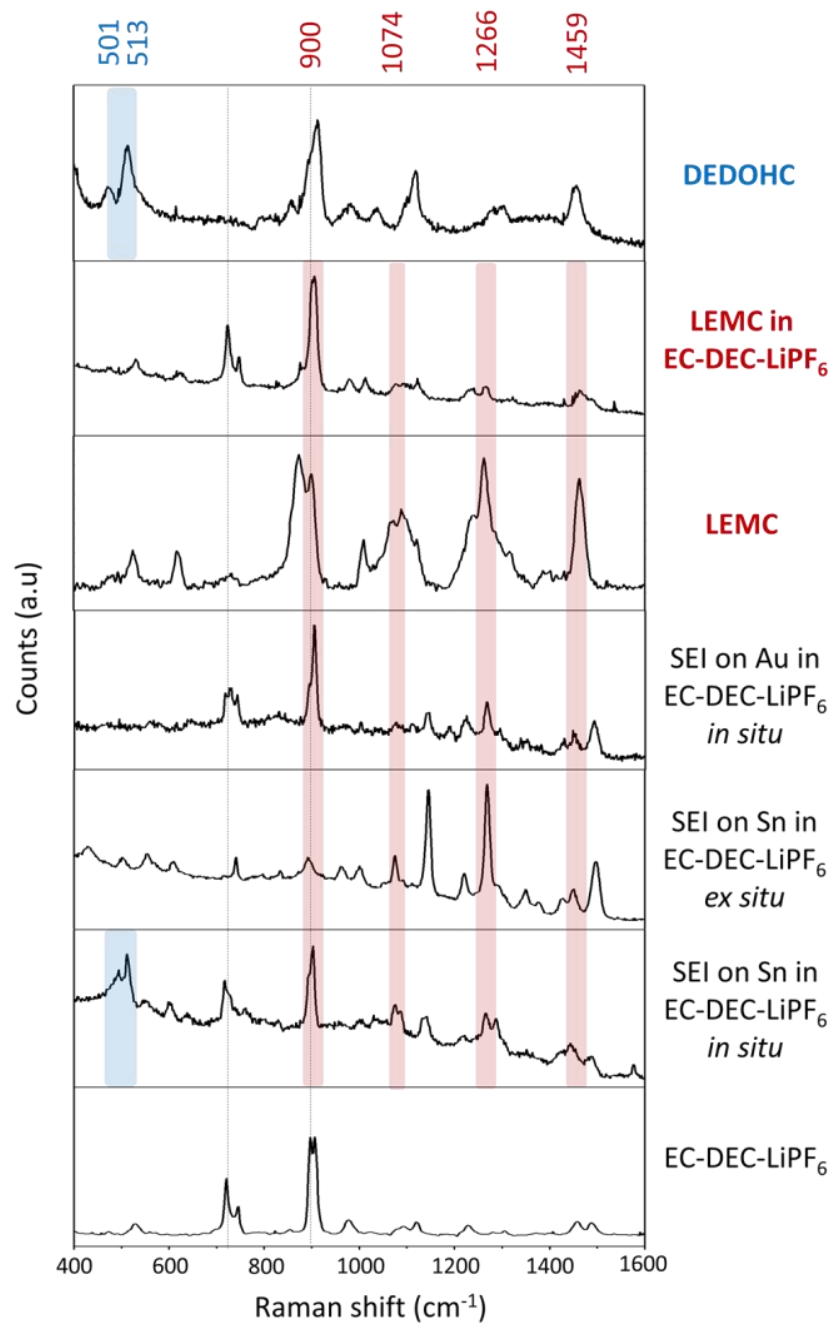

comparison with chemically synthetized $L E D C$. However a recent work by Wang et $\mathrm{al}^{8}$, rediscussed by Freunberger ${ }^{35}$, evidenced that the synthetic route followed by many authors to produced $\angle E D C$ via the intermediate di-lithium alkoxide ( $\mathrm{LiO}$ $\mathrm{CH}_{2}-\mathrm{CH}_{2}-\mathrm{OLi}$ ) actually lead to $\mathrm{LEMC}$ given that the reaction intermediate is not as believed the di- but the monolithiated counterpart. The insulating character of $L E D C$ which contrast with the high $\mathrm{Li}^{+}$conductivity of $L E M C$ additionally supports their claim. In the present study, LEMC is detected (1074 and $1266 \mathrm{~cm}^{-1}$ ) at potential below $1 \mathrm{~V}$ concomitantly with the disappearance of the bands at 1065 and $1285 \mathrm{~cm}^{-1}$, which could support the formation of $L E M C$ following the (electro)chemical evolution of a non-stable compound within the SEI (Figure 4c summarizes the different mechanisms proposed by Wang et $\mathrm{al}^{8}$ which can explain the formation of $L E M C, L E C, L M C$ in $E C$ $D E C L_{i P F_{6}}$ ). As the Raman spectrum of synthetic $L E D C$ shows a strong intensity band at $1090 \mathrm{~cm}^{-1}$ which the position does not seem to be influenced by solvation effect (see Fig. S16), the possible formation of $L E M C$ from the $L E D C$ hydrolysis/protonation $^{8}$ is not evidenced here. It is also important to mention the

(b)
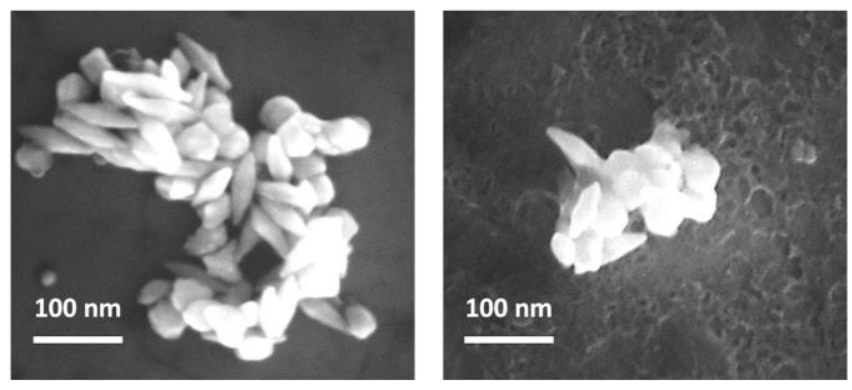

(c)
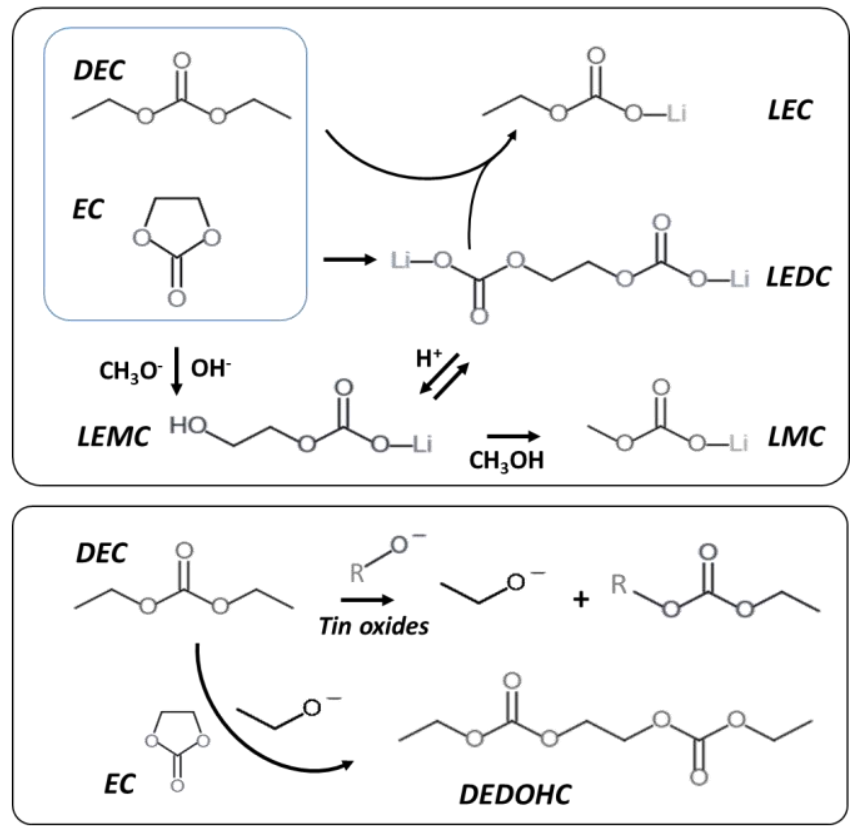

Figure 4: SEI composition and formation mechanisms (a) Comparison of reference Raman spectra of LEMC and DEDOHC with the Raman spectra obtained on gold electrode in situ at $100 \mathrm{mV}$, on tin electrode at $100 \mathrm{mV}$ and ex situ on tin electrode after a $100 \mathrm{mV}$ polarization. The bands highlighted in red and blue correspond respectively to the one of the electrolyte and to the most distinguishable one of DEDOHC. All three experiments were performed in similar conditions in EC/DEC=50/50 (v/v), 1.0 M LiPF 6 electrolyte. (b) SEM micrograph of pristine (left) and cycled (right) tin electrodes (CV in EC-DEC LiPF $)$ decorated with SHIN agglomerates, showing the growth of a rough surface film (SEI) below the particles. (c) Summary of possible electrolyte degradation mechanism to explain the composition of the SEI on tin anodes in contact with EC-DEC LiPF6. 
absence of peaks associated with $L E C$ and $L M C$ (see in SI, Fig. $\mathrm{S} 12 \mathrm{~b}$ and S15), since the formation of $L M C$ and LEC could be facilitated in presence of $E M C$ of $D M C$ in the electrolyte as reported by $\mathrm{Xu}$ et $\mathrm{al}^{36}$ and Wang et al. ${ }^{8}$ Finally, note that in $P C$ based electrolyte, the bands at 1074 and $1266 \mathrm{~cm}^{-1}$ associated with $L E M C$, are absent and instead two bands are present at 924 and $1088 \mathrm{~cm}^{-1}$ which we assigned to Lithium Propylene di-Carbonate $(L P D C)$ (see SI Fig. S17), in agreement with the conclusions of Dedryvère et al. ${ }^{37}$

Formation of electrolyte soluble DEDOHC - The double band at 501-513 $\mathrm{cm}^{-1}$ which appears in situ only on tin anode and not on gold and which almost disappears ex situ after thorough rinsing of the tin electrode with $D E C$, suggests that it can be attributed to the $D E D O H C$ compound. The dynamics of the $501 \mathrm{~cm}^{-1}$ band appearance and disappearance supports this claim as $D E D O H C$ may be produced upon reduction of the electrolyte between 1.6 and $1 \mathrm{~V}$ till the current drops to zero, then diffuses away. Similar dynamical behavior has been observed in previous studies ${ }^{27} 32$ by operando AFM and ellipsometry. $D E D O H C$ has been reported in a series of papers by the groups of Somorjai and Komvopoulos ${ }^{4111}$ using ex situ and in situ IR spectroscopy on different electrode materials $\left(\mathrm{Ni}^{4}, \mathrm{Au}^{4}\right.$ $\left.{ }^{10}, \mathrm{Si}^{11}\right)$ in $E C-D E C \mathrm{LiPF}_{6}$, including tin anode. ${ }^{40}$ Its formation on tin would result from a catalytic process involving surface tin oxides, the formation of alkyl or alkoxide radicals and the reaction with $E C$ and $D E C^{4}$ (Figure 4c), explaining why it is not observed on gold. The specific properties of tin electrode surface promoting the formation of soluble $D E D O H C$ in the electrolyte could account for the large irreversible currents and the delayed passivation of the electrode. Slight change in the linear carbonate structure $E M C$ instead of $D E C$ may limit the formation of $\mathrm{DEDOHC}$ leading to drastic improvement of the passivating ability of tin anodes as shown earlier. Finally SHINERS results, which will be detailed in a following publication, were collected on tin in $P C$ based electrolyte (Fig. S17). In such electrolyte, which do not requires the use of linear carbonate ( $P C$ is liquid at room temperature), the $501 \mathrm{~cm}^{-1}$ band is not observed supporting the $D E D O H C$ formation mechanism proposed in Figure 4c involving both $D E C$ and $E C$.

In summary, SHINERS using $\mathrm{Au} @ \mathrm{SiO}_{2}$ bipyramid nanoparticles (anisotropic $S H I N s$ ) with plasmon absorption in the nearinfrared spectral domain prove to successfully extract within $L I B$ electrolyte the chemical signature of Raman blind molecular monolayer and of passive films (SEI) developed on tin and gold electrodes. Confocal Raman microscopy associated with scanning near-infrared laser allows minimizing the Raman and fluorescence signal of the surrounding $L I B$ organic electrolyte and cancelling out of its photothermal breaking on SHINs upon light exposure. The dynamic tracking of the SEI formation and composition evolution on tin anode in coin-cell filled with $E C$ $D E C L_{i P F}$ is consistent with the recently developed hypothesis that upon reduction of the $E C-D E C$ solvent mixture, $L E M C$ is predominantly formed over the often reported $L E D C$ which is prone to hydrolysis and not clearly identified here. We further back up this idea by evidencing the formation of $L P D C$ (and the absence of $L E M C$ ), upon cycling of tin in cells filled with $P C$ $L i P F_{6}$. Other electrolyte reduction products often reported, e.g. $L E C, L M C, L i F, P E O$ were not clearly identified during the early stage of the $\mathrm{SEI}$ formation, $\mathrm{Li}_{2} \mathrm{CO}_{3}$ proved absent. A Raman signature which shows an interesting dynamics of appearance and disappearance along the reductive potential scan was clearly assigned to the electrolyte soluble $D E D O H C$ compound which forms on tin but not on gold anodes through a catalytic process involving tin oxide, $D E C$ and $E C$. The cyclic formation of $D E D O H C$, concomitant with the repeated emergence upon cycling of the cathodic peak around $1.2 \mathrm{~V}$, accounts for the poor passivating properties of tin in $E C-D E C \mathrm{LiPF}_{6}$ electrolyte and for its large irreversible capacity. Some Raman bands were left unattributed, emphasizing the necessity to explore, depending on the electrode and electrolyte composition, further electrolyte reduction and oxidation paths involving possible $S E I$ or $C E I$ components respectively. The accurate description of the composition of electrolyte interphases the key element behind all $\mathrm{Li}$, $\mathrm{Na}$ or multivalent-ion battery operation and failure, is the prerequisite to the design of new electrolyte and electrode formulations. Similarly to this study, the experimental (and theoretical) chemical signatures of $S E I$ (and $C E I$ ) compounds obtained through chemical synthesis could be gathered within an openaccess online database available to the battery community. Although the SHIN activity is still not fully understood and needs further work (e.g. the origin of the potential dependent signal extinction in $E C-E M L_{i P F_{6}}$ electrolyte), the major optimizations around SHINERS implementation for $L I B$ applications proposed in this study could be used as benchmarks for the characterization of any systems beyond $L I B$ s which operate under harsh conditions.

\section{ASSOCIATED CONTENT}

The Supporting information includes the experimental details and seventeen figures illustrating:

- The optimization of the SHINERS measurements for LIB applications (S1 to S5)

- The full set of data of operando SHINERS measurements in $L I B$ electrolyte (S6 to S10)

- The Raman signature assignment covering synthesis protocols and Raman signature of synthetic SEI compounds (S11 to $\mathrm{S} 17)$.

\section{AUTHOR INFORMATION}

\section{Corresponding Author}

Ivan T. Lucas - Sorbonne Université, CNRS, Laboratoire LISE, 75000 Paris, France - Email: ivan.lucas@sorbonne-universite.fr

\section{Author Contributions}

A.G. produced all the Raman and electrochemical data presented in this work. C.L. and L.F. synthesized the LEMC, LEDC, $L P D C$, $L M C, L E C$ organic compounds and analyzed their NMR spectra. L.F., B.T.B., A.G. and I.L. developed the protocol for the synthesis of 90 and $120 \mathrm{~nm}$ isotrope $\mathrm{SHIN}$ objects ( $\mathrm{Au} @ \mathrm{SiO}_{2}$ spherical nanoparticles). L.F., A.G. and I.L. developed the protocol for the synthesis of 70 and $140 \mathrm{~nm}$ anisotrope SHIN objects ( $\mathrm{Au} @ \mathrm{SiO}_{2}$ bipyramid nanoparticles) for red and near-infrared Raman probes. I.T.L. and A.G. developed the spectro-electrochemical coin-cells and SHINERS analyses using scanning Raman probes. I.T.L. and J.D. led this study. A.G., C.L., B.T.B., L.F., J.D. and I.T.L. wrote this manuscript and contributed to discussion.

\section{Funding Sources}

This project was funded by the battery manufacturer Saft (a company of Total), the ANRT (CIFRE $n^{\circ} 2018 / 1579$ ) and by the ANRCarLIB (grant numbers: ANR-15-CE05-0002).

\section{ACKNOWLEDGMENT}

We would like to thanks J.-M. Siaugue and A. Michel from PHENIX lab for fruitful discussion on core-shell particle synthesis, L. Boudinet and C. Bignaud for their bachelor and master internship work respectively preceding this study, F. Pillier in LISE lab for the electron microscopy analyses.

\section{REFERENCES}


(1) Armand, M.; Tarascon, J.-M. Building Better Batteries. Nature 2008, 451 (7179), 652-657.

(2) Peled, E. The Electrochemical Behavior of Alkali and Alkaline Earth Metals in Nonaqueous Battery Systems - The Solid Electrolyte Interphase Model. J. Electrochem. Soc. 1979, 126 (12), 2047-2051.

(3) Edström, K.; Gustafsson, T.; Thomas, J. O. The CathodeElectrolyte Interface in the Li-Ion Battery. Electrochimica Acta 2004, 50 (2-3), 397-403.

(4) Shi, F.; Zhao, H.; Liu, G.; Ross, P. N.; Somorjai, G. A.; Komvopoulos, K. Identification of Diethyl 2,5-Dioxahexane Dicarboxylate and Polyethylene Carbonate as Decomposition Products of Ethylene Carbonate Based Electrolytes by Fourier Transform Infrared Spectroscopy. J. Phys. Chem. C 2014, 118 (27), 14732-14738.

(5) Tu, Z.; Choudhury, S.; Zachman, M. J.; Wei, S.; Zhang, K.; Kourkoutis, L. F.; Archer, L. A. Designing Artificial SolidElectrolyte Interphases for Single-Ion and High-Efficiency Transport in Batteries. Joule 2017, 1 (2), 394-406.

(6) Verma, P.; Maire, P.; Novák, P. A Review of the Features and Analyses of the Solid Electrolyte Interphase in Li-Ion Batteries. Electrochimica Acta 2010, 55 (22), 6332-6341.

(7) Heiskanen, S. K.; Kim, J.; Lucht, B. L. Generation and Evolution of the Solid Electrolyte Interphase of Lithium-Ion Batteries. Joule 2019, 3 (10), 2322-2333.

(8) Wang, L.; Menakath, A.; Han, F.; Wang, Y.; Zavalij, P. Y.; Gaskell, K. J.; Borodin, O.; Iuga, D.; Brown, S. P.; Wang, C.; $\mathrm{Xu}, \mathrm{K}$; Eichhorn, B. W. Identifying the Components of the Solid-Electrolyte Interphase in Li-Ion Batteries. Nat. Chem. 2019, 11 (9), 789-796.

(9) Zhang, J.-N.; Li, Q.; Wang, Y.; Zheng, J.; Yu, X.; Li, H. Dynamic Evolution of Cathode Electrolyte Interphase (CEI) on High Voltage LiCoO2 Cathode and Its Interaction with Li Anode. Energy Storage Materials 2018, 14, 1-7.

(10) Shi, F.; Ross, P. N.; Zhao, H.; Liu, G.; Somorjai, G. A.; Komvopoulos, K. A Catalytic Path for Electrolyte Reduction in Lithium-Ion Cells Revealed by in Situ Attenuated Total Reflection-Fourier Transform Infrared Spectroscopy. J. Am. Chem. Soc. 2015, 137 (9), 3181-3184.

(11) Shi, F.; Ross, P. N.; Somorjai, G. A.; Komvopoulos, K. The Chemistry of Electrolyte Reduction on Silicon Electrodes Revealed by in Situ ATR-FTIR Spectroscopy. J. Phys. Chem. C 2017, 121 (27), 14476-14483.

(12) Buqa, H.; Würsig, A.; Vetter, J.; Spahr, M. E.; Krumeich, F.; Novák, P. SEI Film Formation on Highly Crystalline Graphitic Materials in Lithium-Ion Batteries. Journal of Power Sources 2006, 153 (2), 385-390.

(13) Horowitz, Y.; Han, H.-L.; Ross, P. N.; Somorjai, G. A. In Situ Potentiodynamic Analysis of the Electrolyte/Silicon Electrodes Interface Reactions - A Sum Frequency Generation Vibrational Spectroscopy Study. J. Am. Chem. Soc. 2016, 138 (3), 726-729.

(14) Baddour-Hadjean, R.; Pereira-Ramos, J.-P. Raman Microspectrometry Applied to the Study of Electrode Materials for Lithium Batteries. Chem. Rev. 2010, 110 (3), 1278-1319.

(15) Willets, K. A.; Van Duyne, R. P. Localized Surface Plasmon Resonance Spectroscopy and Sensing. Annu. Rev. Phys. Chem. 2007, 58 (1), 267-297.

(16) Li, H.; Mo, Y.; Pei, N.; Xu, X.; Huang, X.; Chen, L. Surface-Enhanced Raman Scattering Study on Passivating Films of Ag Electrodes in Lithium Batteries $\uparrow$. J. Phys. Chem. B 2000, 104 (35), 8477-8480.

(17) Li, G.; Li, H.; Mo, Y.; Chen, L.; Huang, X. Further Identification to the SEI ${ }^{\circledR} 1 \mathrm{~m}$ on $\mathrm{Ag}$ Electrode in Lithium Batteries by Surface Enhanced Raman Scattering (SERS). Journal of Power Sources 2002, 104(2), 190-194.

(18) Ha, Y.; Tremolet de Villers, B. J.; Li, Z.; Xu, Y.; Stradins, P.; Zakutayev, A.; Burrell, A.; Han, S.-D. Probing the Evolution of Surface Chemistry at the Silicon-Electrolyte Interphase via In Situ Surface-Enhanced Raman Spectroscopy. J. Phys. Chem. Lett. 2020, 11 (1), 286-291.

(19) Nanda, J.; Yang, G.; Hou, T.; Voylov, D. N.; Li, X.; Ruther, R. E.; Naguib, M.; Persson, K.; Veith, G. M.; Sokolov, A. P. Unraveling the Nanoscale Heterogeneity of Solid Electrolyte Interphase Using Tip-Enhanced Raman Spectroscopy. Joule 2019, 3 (8), 2001-2019.

(20) Ayache, M.; Jang, D.; Syzdek, J.; Kostecki, R. Near-Field IR Nanoscale Imaging of the Solid Electrolyte Interphase on a HOPG Electrode. J. Electrochem. Soc. 2015, 162 (13), A7078A7082.

(21) Kurouski, D.; Mattei, M.; Van Duyne, R. P. Probing Redox Reactions at the Nanoscale with Electrochemical Tip-Enhanced Raman Spectroscopy. Nano Lett. 2015, 15 (12), 7956-7962.

(22) Touzalin, T.; Joiret, S.; Lucas, I. T.; Maisonhaute, E. Electrochemical Tip-Enhanced Raman Spectroscopy Imaging with $8 \mathrm{Nm}$ Lateral Resolution. Electrochemistry Communications 2019, 108,

(23) Li, J. F.; Huang, Y. F.; Ding, Y.; Yang, Z. L.; Li, S. B.; Zhou, X. S.; Fan, F. R.; Zhang, W.; Zhou, Z. Y.; Wu, D. Y.; Ren, B.; Wang, Z. L.; Tian, Z. Q. Shell-Isolated NanoparticleEnhanced Raman Spectroscopy. Nature 2010, 464 (7287), 392 395.

(24) Hy, S.; Felix; Chen, Y.-H.; Liu, J.; Rick, J.; Hwang, B.-J. In Situ Surface Enhanced Raman Spectroscopic Studies of Solid Electrolyte Interphase Formation in sodiu Ion Battery Electrodes. Journal of Power Sources 2014, 256, 324-328.

(25) Cabo-Fernandez, L.; Bresser, D.; Braga, F.; Passerini, S.; Hardwick, L. J. In-Situ Electrochemical SHINERS Investigation of SEI Composition on Carbon-Coated Zn 0.9 Fe $0.1 \mathrm{O}$ Anode for Lithium-Ion Batteries. Batteries \& Supercaps 2019, 2 (2), 168-177.

(26) Li, C.-Y.; Yu, Y.; Wang, C.; Zhang, Y.; Zheng, S.-Y.; Li, J.-F.; Maglia, F.; Jung, R.; Tian, Z.-Q.; Shao-Horn, Y. Surface Changes of LiNi x Mn y Co $1-\mathrm{x}-\mathrm{y}$ O 2 in Li-Ion Batteries Using in Situ Surface-Enhanced Raman Spectroscopy. J. Phys. Chem. C 2020, 124 (7), 4024-4031.

(27) Lucas, I. T.; Pollak, E.; Kostecki, R. In Situ AFM Studies of SEI Formation at a Sn Electrode. Electrochemistry Communications 2009, 11 (11), 2157-2160.

(28) Beaulieu, L. Y.; Beattie, S. D.; Hatchard, T. D.; Dahn, J. R. The Electrochemical Reaction of Lithium with Tin Studied By In Situ AFM. J. Electrochem. Soc. 2003, 150 (4), A419.

(29) Sánchez-Iglesias, Ana, Naomi Winckelmans, Thomas Altantzis, Sara Bals, Marek Grzelczak, et Luis M. Liz-Marzán. 2017. « High-Yield Seeded Growth of Monodisperse Pentatwinned Gold Nanoparticles through Thermally Induced Seed Twinning ». Journal of the American Chemical Society 139 (1): 107-10.

(30) Liu, M.; Guyot-Sionnest, P. Mechanism of Silver(I)-Assisted Growth of Gold Nanorods and Bipyramids. J. Phys. Chem. B 2005, 109 (47), 22192-22200.

(31) Ou, W.; Zhou, B.; Shen, J.; Lo, T. W.; Lei, D.; Li, S.; Zhong, J.; Li, Y. Y.; Lu, J. Thermal and Nonthermal Effects in Plasmon-Mediated Electrochemistry at Nanostructured Ag Electrodes. Angew. Chem. Int. Ed. 2020, 59 (17), 6790-6793. 
(32) Lucas, I. T.; Syzdek, J.; Kostecki, R. Interfacial Processes at Single-Crystal $\beta$-Sn Electrodes in Organic Carbonate Electrolytes. Electrochemistry Communications 2011, 13 (11), 1271-1275.

(33) Aurbach, D.; Moshkovich, M.; Cohen, Y.; Schechter, A. The Study of Surface Film Formation on Noble-Metal Electrodes in Alkyl Carbonates/Li Salt Solutions, Using Simultaneous in Situ AFM, EQCM, FTIR, and EIS. Langmuir 1999, 15 (8), 2947-2960.

(34) Aurbach, D.; Markovsky, B.; Shechter, A.; Ein-Eli, Y.; Cohen, H. A Comparative Study of Synthetic Graphite and Li Electrodes in Electrolyte Solutions Based on Ethylene Carbonate-Dimethyl Carbonate Mixtures. J. Electrochem. Soc. 1996, 143 (12), 3809-3820.

(35) Freunberger, S. A. Interphase Identity Crisis. Nat. Chem. 2019, 11 (9), 761-763.

(36) Xu, Kang, Guorong V. Zhuang, Jan L. Allen, Unchul Lee, Sheng S. Zhang, Philip N. Ross, T. Richard Jow. Syntheses and Characterization of Lithium Alkyl Mono- and Dicarbonates as Components of Surface Films in Li-Ion Batteries. The Journal of Physical Chemistry B 2006, 110 (15): 7708-19.

(37) Dedryvère, R.; Gireaud, L.; Grugeon, S.; Laruelle, S.; Tarascon, J.-M.; Gonbeau, D. Characterization of Lithium Alkyl Carbonates by X-Ray Photoelectron Spectroscopy: Experimental and Theoretical Study. J. Phys. Chem. B 2005, 109 (33), $15868-15875$. 